INDIAN JOURNAL OF POWER \& RIVER VALLEY DEVELOPMENT www.ijprvd.info

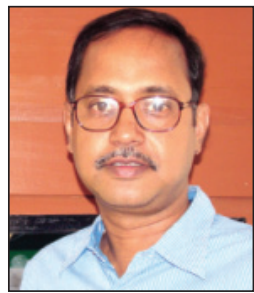

\title{
Greening and biodiversity protection in the power sector: benefits outweigh cost
}

Natural environments are important determinants of human health and wellbeing. A range of mental and physical health benefits are associated with 'greenness', where greenness is an aspect of the natural environment that corresponds to vegetation cover. Higher levels of residential greenness have been associated with better mental health in adults, higher birth weight, reduced cardiovascular disease mortality, and better general health. Blue spaces, which include all visible surface water are another part of nature which preliminary evidence has suggested may also be important for health.
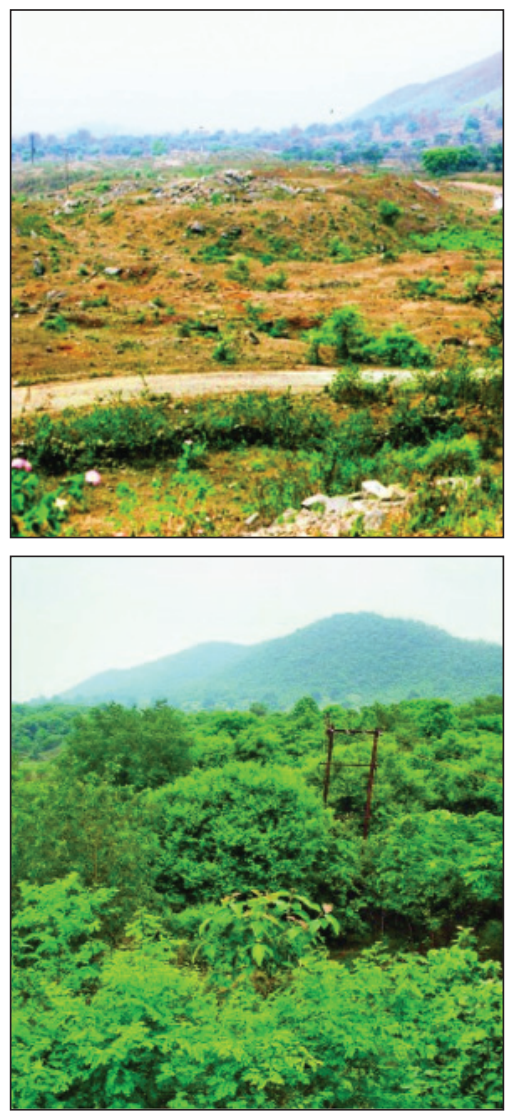

A forest re-grown in a mining area
Biodiversity, which includes all life forms, ranges from the diversity of genes present in a location to counts and turnover of species in whole ecosystems. Increasingly, researchers are suggesting biodiversity is positively linked to psychological and mental health outcomes, and also improved health via immune system development and regulation as a result of exposure to environmental microbiota. However, while there is preliminary evidence indicating that biodiversity may be beneficial to health, the body of evidence is sparse.

A study on English adults examined the relationships between subjective wellbeing and three types of nature exposure neighborhood exposure to nature (measured as green space and coastal proximity), nature visit frequency over the last 12 months, and visiting a natural environment yesterday.

A recent study in Australia concludes that natural features of the environment such as greenness and biodiversity are important determinants of subjective wellbeing. Taken with other health benefits of natural environments, this further supports the case for considering and integrating biodiversity and nature into both urban planning and health policies and practices. The study makes a difference in contribution between public green (the greening by the side of the public road, green belt/cover, water bodies) and private green (house lawns, garden, balcony garden and roof top garden). The study showed, though not a proof till now, that private greenness was the main component of overall greenness and was more strongly associated with subjective wellbeing than public greenness. Mines can incorporate both in an ever-increasing manner so that the employee and family health, and performance improve. 